JOURNAL OF INTEGRAL EQUATIONS AND APPLICATIONS

Volume 9, Number 4, Fall 1997

\title{
POSITIVE SOLUTIONS OF SUPERLINEAR HAMMERSTEIN INTEGRAL EQUATIONS IN BANACH SPACES
}

\author{
BENDONG LOU
}

ABSTRACT. Some existence results of positive solutions for superlinear Hammerstein integral equations in Banach spaces are obtained by means of the fixed point index theory. Some applications to superlinear Sturm-Liouville problems in Banach spaces are given. We do not use the method of prior estimate which is used in many papers for similar problems. Our theorems extend some former results in this field.

1. Introduction. Let $E$ be a Banach space. Consider the following Hammerstein integral equation in $E$ :

$$
\varphi(t)=\int_{0}^{1} k(t, s) f(s, \varphi(s)) d s \equiv A \varphi(t),
$$

where $I=[0,1], k(t, s) \in C\left[I \times I, R^{1}\right]$ is nonnegative, $f \in C[I \times E, E]$ and $\varphi \in C[I, E]$. In this paper we get some existence theorems of positive solutions of equation (1) by means of the fixed point index theory and then give some applications to superlinear Sturm-Liouville problems in Banach spaces.

Many authors have studied the existence of nontrivial solutions for two-point boundary value problems (BVPs) of ordinary differential equations, cf. $[5,8,3,6]$. In $[5,8,3]$, the authors investigated the superlinear Sturm-Liouville problems. In [5], the superlinear condition is expressed as

$$
\lim _{\substack{u \in P \\\|u\| \rightarrow+\infty}} \frac{\phi(f(t, u))}{\phi(u)}=+\infty
$$

$$
\text { uniformly in } t \in[\alpha, \beta] \subset(0,1) \text {, }
$$

where $P$ is a cone of $E$ and $\phi$ is a positive linear functional. Clearly this superlinear condition is not very sharp since the righthand side of

\footnotetext{
Received by the editors on June 5, 1996, and in revised form on May 10, 1996.

Project supported by the National Natural Science Foundation of China and the State Education Commission Doctoral Foundation of China.
}

Copyright (C)1997 Rocky Mountain Mathematics Consortium 
it is $+\infty$. In [8], the superlinear condition is expressed by an order relation

$$
f(t, u) \geq a(t) u-b(t), \quad \forall t \in I, u \in P,
$$

where $a \in C(I)$ with $a(t)>0$ and $b \in C[I, E]$. This condition is sharp but the results for Sturm-Liouville problems cannot be extended to integral equations easily, see $[\mathbf{8}]$. The superlinear condition in $[\mathbf{3}]$ is similar to that in $[\mathbf{8}]$, and the BVPs considered in $[\mathbf{3}]$ is a scalar one. Furthermore, $[\mathbf{3}]$ used the method of prior estimate which is also used in [6] and is complicated. In this paper we consider the Hammerstein integral equation (1) instead of BVPs. Our superlinear condition is also expressed by an order relation, see $\left(H_{1}\right)$ in Section 3, and is sharp. We do not use the method of prior estimate. Our results generalize some of the results in $[\mathbf{5}, \mathbf{8}, \mathbf{3}]$.

In what follows, let $P$ be a normal cone of $E$ with a normal constant $N$, see $[\mathbf{1}, \mathbf{2}] . \quad \theta \leq u \leq v$ implies $\|u\| \leq N\|v\|$, where $\theta$ is the zero element of $E$. It is clear that $C[I, E]$ is a Banach space with norm $\|\varphi\|_{C}=\max _{t \in I}\|\varphi(t)\|, \varphi \in C[I, E]$. Let $K=\{\varphi \in C[I, E] \mid \varphi(t) \in P$ for all $t \in I\}$, then $K$ is a cone of $C[I, E] . \varphi \in C[I, E]$ is called a positive solution of equation (1) if it satisfies (1) and $\varphi \in K \backslash\{\theta\}$. The closed ball in $E$ is denoted by $T_{l}=\{u \in E \mid\|u\| \leq l\}, l>0$, and the open ball in $C[I, E]$ is denoted by $B_{l}=\left\{\varphi \in C[I, E] \mid\|\varphi\|_{C}<l\right\}$, $l>0$.

2. Some lemmas. Let $k(t, s) \in C\left[I \times I, R^{1}\right]$ be defined as in (1) and $h(t) \in C(I)$. Define operators $K, H: C(I) \rightarrow C(I)$ by

$$
\begin{aligned}
& K x(t)=\int_{0}^{1} k(t, s) x(s) d s, \\
& H x(t)=h(t) x(t), \quad x \in C(I) .
\end{aligned}
$$

Lemma 1. Let $h(t)>0$ almost everywhere $t \in I$. If $k(t, s)$ satisfies one of the following two conditions:

(i) $k(t, s)$ is nonnegative, continuous and $k(t, t) \not \equiv 0, t \in I$;

(ii) $k(t, s)$ is nonnegative, continuous and symmetric and $k(t, s) \not \equiv 0$, $t, s \in I$. 
Then $H K: C(I) \rightarrow C(I)$ is a linear, completely continuous positive operator and the spectral radius of $H K, r(H K) \equiv r$, is positive.

Proof. It is clear that $K: C(I) \rightarrow C(I)$ is completely continuous and $H: C(I) \rightarrow C(I)$ is a linear, bounded positive operator. Therefore, $K H, H K: C(I) \rightarrow C(I)$ are linear, completely continuous positive operators. We now prove that $r(H K)>0$.

We first suppose that (i) is satisfied. Since $k(t, t) \not \equiv 0, t \in I$, there exists $t_{0} \in I$ such that $k\left(t_{0}, t_{0}\right)>0$. On account of the continuity of $k$, there exist a small interval $N_{\delta_{1}}\left(t_{0}\right)=\left\{t|t \in I| t-,t_{0} \mid<\delta_{1}\right\}$ and a constant $\varepsilon>0$ such that $k(t, s) \geq \varepsilon>0$ for all $t, s \in N_{\delta_{1}}\left(t_{0}\right)$. Since $h(t)>0$ almost everywhere $t \in I$ and $h$ is continuous, there exist a small interval $N_{2 \delta}\left(t_{1}\right)=\left\{t\left|t \in N_{\delta_{1}}\left(t_{0}\right),\right| t-t_{1} \mid<2 \delta\right\} \neq \varnothing$ and a constant $\varepsilon_{1}>0$ such that $h(t) \geq \varepsilon_{1}>0$ for all $t \in N_{2 \delta}\left(t_{1}\right)$.

Define a real-valued function $x_{0}(t) \in C(I)$ by

$$
x_{0}(t)= \begin{cases}1 & \text { if } t \in N_{\delta}\left(t_{1}\right), \\ 0 & \text { if } t \notin N_{2 \delta}\left(t_{1}\right), \\ \text { numbers between } 0 \text { and } 1 & \text { if } t \in N_{2 \delta}\left(t_{1}\right) \backslash N_{\delta}\left(t_{1}\right) .\end{cases}
$$

Then, for $t \in N_{2 \delta}\left(t_{1}\right)$, we have

$$
\begin{aligned}
K H x_{0}(t) & =\int_{0}^{1} k(t, s) h(s) x_{0}(s) d s \\
& \geq \int_{N_{\delta}\left(t_{1}\right)} k(t, s) h(s) x_{0}(s) d s \\
& \geq \varepsilon \varepsilon_{1} \delta \geq \varepsilon \varepsilon_{1} \delta x_{0}(t) .
\end{aligned}
$$

By the definition of $x_{0}(t)$, we have

$$
K H x_{0}(t) \geq \varepsilon \varepsilon_{1} \delta x_{0}(t), \quad t \in I .
$$

Let $P_{0}=\{x \in C(I) \mid x(t) \geq 0$ for all $t \in I\}$. Then $P_{0}$ is a cone of $C(I)$ and $x_{0} \in P_{0} \backslash\{\theta\}$. Since $K H$ is a linear completely continuous positive operator, it follows from (2) and Corollary 2.1 in [9] that $r(K H) \geq \varepsilon \varepsilon_{1} \delta>0$. Consequently, by the Krein-Rutman theorem there exists $x_{1} \in P_{0} \backslash\{\theta\}$ such that $K H x_{1}=r(K H) x_{1}$, and 
so $H K\left(H x_{1}\right)=r(K H)\left(H x_{1}\right)$. Clearly, $H x_{1}(t)=h(t) x_{1}(t) \in P_{0} \backslash\{\theta\}$, therefore $r(H K) \geq r(K H)>0$.

If case (ii) holds, we can construct a function $x_{0} \in P_{0} \backslash\{\theta\}$ in a similar way such that $K H x_{0}(t) \geq \varepsilon x_{0}(t), t \in I$, for some $\varepsilon>0$. Then a similar discussion as above shows that $r(H K)>0$. This completes the proof.

Remark 1. Let $K$ and $H$ be as above, and let $L: C(I) \rightarrow C(I)$ be defined by

$$
L x(t)=\int_{0}^{1} k(s, t) h(s) x(s) d s .
$$

Then it is not difficult to verify that $r(L)=r(H K)$. So if $k$ and $h$ satisfy all the conditions of Lemma 1, then $r(L)=r(H K)>0$.

3. Main theorems. We shall use the following conditions:

$\left(H_{0}\right) \quad f \in C[I \times P, P]$. For any $l>0, f$ is uniformly continuous on $I \times\left(P \cap T_{l}\right)$, and there exists a constant $L_{l}$ with $0 \leq L_{l}<1 /(2 M)$ such that

$$
\alpha(f(t, D)) \leq L_{l} \alpha(D), \quad t \in I, D \subset P \cap T_{l},
$$

where $M=\max _{t, s \in I} k(t, s)>0$ and $\alpha(\cdot)$ denotes the Kuratowski measure of noncompactness.

$\left(H_{1}\right)$ There exist $R>0$ and $h(t) \in C(I)$ such that

$$
f(t, u) \geq r^{-1} h(t) u, \quad t \in I, u \in P, \quad\|u\| \geq R,
$$

where $r=r(H K)$ is defined as in Lemma 1.

$\left(H_{2}\right)$ There exist $R_{0}>0, b(t) \in C(I), b(t)>0, t \in I$, such that

$$
\|f(t, u)\| \leq \lambda_{0} b(t)\|u\|, \quad t \in I, u \in P, \quad\|u\| \leq R_{0},
$$

where $\lambda_{0} \geq 0$ is the first eigenvalue of

$$
x(t)=\lambda \int_{0}^{1} k(t, s) b(s) x(s) d s \equiv \lambda B x(t), \quad x \in C(I) .
$$

$\left(H_{3}\right)$ There exist $R_{0}>0$ and $h(t) \in C(I)$ such that

$$
f(t, u) \geq r^{-1} h(t) u, \quad t \in I, u \in P, \quad\|u\| \leq R_{0} .
$$


$\left(H_{4}\right)$ There exists $\eta>0$ such that

$$
\sup _{\substack{t \in I \\ u \in P \cap T_{\eta}}}\|f(t, u)\|<\frac{\eta}{M N} .
$$

Our main result is:

Theorem 1. Assume that $k$ and $h$ satisfy all the conditions of Lemma 1 and $k(t, s) \geq \varepsilon k(\tau, s), t, \tau, s \in I$ for some $\varepsilon>0$. Suppose that $\left(H_{0}\right),\left(H_{1}\right)$ and $\left(H_{2}\right)$ are satisfied. Then equation (1) has at least one positive solution in $C[I, E]$.

Proof. By $\left(H_{0}\right)$ and Lemma 2 in [5], we know that $A: K \cap \bar{B}_{l} \rightarrow K$ is a $k_{l}$-set-contraction with $k_{l}<1$ for any $l>0$. Set $Q=\{\varphi \in K$ $\varphi(t) \geq \varepsilon \varphi(s), t, s \in I\}$; clearly, $Q \neq \phi$ is also a cone of $C[I, E]$. Since $k(t, s) \geq \varepsilon k(\tau, s), t, s, \tau \in I$ and $f \in C[I \times P, P]$, we have for any $\varphi \in K$,

$$
\begin{aligned}
A \varphi(t) & =\int_{0}^{1} k(t, s) f(s, \varphi(s)) d s \\
& \geq \varepsilon \int_{0}^{1} k(\tau, s) f(s, \varphi(s)) d s \\
& =\varepsilon A \varphi(\tau), \quad t, \tau \in I,
\end{aligned}
$$

i.e., $A \varphi \in Q$. Thus,

$$
A K \subset Q .
$$

By Lemma 1, $H K: C(I) \rightarrow C(I)$ is a linear, completely continuous positive operator and $r(H K)>0$. By Remark 1, we know that $L: C(I) \rightarrow C(I)$ is also a linear, completely continuous positive operator and $r(L)=r(H K)>0$. Then it follows from the KreinRutman theorem, see [9], that there exists $p(t) \in C(I)$ with $p(t) \geq 0$, $p(t) \not \equiv 0$, such that

$$
L p(t)=r(L) p(t)=r p(t) .
$$

Choose a $u_{0} \in P \backslash\{\theta\}$ and $R_{1}>R_{0}$ such that $R_{1} \geq N R / \varepsilon$, where $N$ is the normal constant of $P$. We assert that

$$
\begin{aligned}
\varphi & \neq A \varphi+\lambda u_{0}, \quad \varphi \in K, \\
\|\varphi\|_{C} & =R_{1}, \quad \lambda \geq 0 .
\end{aligned}
$$


In fact, if there exist $\varphi_{1} \in K,\left\|\varphi_{1}\right\|_{C}=R_{1}$ and $\lambda_{1} \geq 0$ such that $\varphi_{1}=A \varphi_{1}+\lambda_{1} u_{0}$, then by (4) and $u_{0} \in Q$ we have $\varphi_{1} \in Q$, i.e., $\varphi_{1}(s) \geq \varepsilon \varphi_{1}(\tau), s, \tau \in I$; thus $\left\|\varphi_{1}(s)\right\| \geq \varepsilon\left\|\varphi_{1}(\tau)\right\| / N, s, \tau \in I$. Since $\tau \in I$ is arbitrary, we have

$$
\left\|\varphi_{1}(s)\right\| \geq \frac{\varepsilon\left\|\varphi_{1}\right\|_{C}}{N}=\frac{\varepsilon R_{1}}{N} \geq R, \quad s \in I .
$$

Without loss of generality, suppose that $\lambda_{1}>0$. Then by $(7)$ and $\left(H_{1}\right)$, we have

$$
\begin{aligned}
h(t) \varphi_{1}(t) & =h(t) A \varphi_{1}(t)+\lambda_{1} h(t) u_{0} \\
& >h(t) \int_{0}^{1} k(t, s) f\left(s, \varphi_{1}(s)\right) d s \\
& \geq r^{-1} h(t) \int_{0}^{1} k(t, s) h(s) \varphi_{1}(s) d s, \quad \text { a.e. } t \in I,
\end{aligned}
$$

which implies by (5) that

$$
\begin{aligned}
\int_{0}^{1} p(t) h(t) \varphi_{1}(t) d t & >r^{-1} \int_{0}^{1} p(t) h(t) d t \int_{0}^{1} k(t, s) h(s) \varphi_{1}(s) d s \\
& =r^{-1} \int_{0}^{1} h(s) \varphi_{1}(s) d s \int_{0}^{1} k(t, s) h(t) p(t) d t \\
& =\int_{0}^{1} p(s) h(s) \varphi_{1}(s) d s
\end{aligned}
$$

a contradiction. Consequently, (6) is true and by the homotopy invariance of the fixed point index, cf. $[\mathbf{1}]$, it is easy to get

$$
i\left(A, K \cap B_{R_{1}}, K\right)=0 .
$$

By the proof of Lemma 1 and $\left(H_{2}\right)$ we know that the operator $B: C(I) \rightarrow C(I)$ defined by (3) is a linear, completely continuous positive operator and $\lambda_{0}>0, r(B)=1 / \lambda_{0}$. Without loss of generality, assume that $\varphi \neq A \varphi$ for all $\varphi \in K,\|\varphi\|_{C}=R_{0}$.

We now prove that

$$
\begin{aligned}
\varphi & \neq \mu A \varphi, \quad \varphi \in K, \\
\|\varphi\|_{C} & =R_{0}, \quad \mu \in I .
\end{aligned}
$$


In fact, if there exist $\varphi_{2} \in K,\left\|\varphi_{2}\right\|_{C}=R_{0}$ and $\mu_{1} \in I$ such that $\varphi_{2}=\mu_{1} A \varphi_{2}$, then $\mu_{1} \in(0,1)$ and, by $\left(H_{2}\right)$,

$$
\begin{aligned}
\left\|\varphi_{2}(t)\right\| & =\mu_{1}\left\|\int_{0}^{1} k(t, s) f\left(s, \varphi_{2}(s)\right) d s\right\| \\
& \leq \mu_{1} \lambda_{0} \int_{0}^{1} k(t, s) b(s)\left\|\varphi_{2}(s)\right\| d s \\
& =\mu_{1} \lambda_{0} B\left(\left\|\varphi_{2}(t)\right\|\right),
\end{aligned}
$$

which implies by Theorem 2.1 in Chapter 5 in $[7]$ that $r(B) \geq$ $\left(\mu_{1} \lambda_{0}\right)^{-1}>1 / \lambda_{0}$, in contradiction with $r(B)=1 / \lambda_{0}$. Consequently, (9) holds and

$$
i\left(A, K \cap B_{R_{0}}, K\right)=1 .
$$

Finally, it follows from $0<R_{0}<R_{1},(8),(10)$ and the additivity of the fixed point index, cf. [1], that

$$
i\left(A, K \cap\left(B_{R_{1}} \backslash \bar{B}_{R_{0}}\right), K\right)=-1,
$$

which implies that $A$ has at least one fixed point in $K \cap\left(B_{R_{1}} \backslash \bar{B}_{R_{0}}\right)$. This completes the proof.

In Theorem 1 , let $h(t) \equiv 1, t \in I$, and $b(t) \equiv 1, t \in I$, then we get

Corollary 1. Suppose that $k$ satisfies all the conditions of Theorem 1 , and $f$ satisfies $\left(H_{0}\right)$. Suppose also that there exist $R>0$, $R_{0}>0$ such that

$$
\begin{aligned}
f(t, u) & \geq r^{-1} u, \quad t \in I, u \in P \\
\|u\| & \geq R
\end{aligned}
$$

and

$$
\begin{aligned}
\|f(t, u)\| & \leq r^{-1}\|u\|, \quad t \in I, u \in P, \\
\|u\| & \leq R_{0},
\end{aligned}
$$

where $r=r(K)>0$. Then equation (1) has at least one positive solution in $C[I, E]$. 
Remark 2. Our superlinear conditions $\left(H_{1}\right)$ and (11) are expressed by the first eigenvalue $r^{-1}$ and what we studied are integral equations, so our results are more general and more essential than some former results, cf. $[\mathbf{5}, \mathbf{8}, \mathbf{3}]$.

Theorem 2. Suppose that $k$ and $h$ satisfy all the conditions of Theorem 1 and $\left(H_{0}\right),\left(H_{1}\right),\left(H_{3}\right)$ and $\left(H_{4}\right)$ are satisfied. Then equation (1) has at least two positive solutions in $C[I, E]$.

Proof. Choose $0<R_{2}<\min \left\{R_{0}, \eta\right\}$ and $R_{1}>\eta$ such that $R_{1}>$ $N R / \varepsilon$. From the proof of Theorem 1 we know that $A: K \cap \bar{B}_{l} \rightarrow K$ is a strict-set contraction for any $l>0$. Thus, by $\left(H_{1}\right)$ and $\left(H_{3}\right)$, in the same way as establishing (8), we can get

$$
i\left(A, K \cap B_{R_{1}}, K\right)=i\left(A, K \cap B_{R_{2}}, K\right)=0 .
$$

On the other hand, we have

$$
\begin{gathered}
A \varphi \nsupseteq \varphi, \quad \varphi \in K, \\
\|\varphi\|_{C}=\eta .
\end{gathered}
$$

In fact, if there exist $\varphi_{0} \in K$ with $\left\|\varphi_{0}\right\|_{C}=\eta$ such that $A \varphi_{0} \geq \varphi_{0}$, then

$$
\begin{aligned}
\theta & \leq \varphi_{0}(t) \leq \int_{0}^{1} k(t, s) f\left(s, \varphi_{0}(s)\right) d s \\
& \leq M \int_{0}^{1} f\left(s, \varphi_{0}(s)\right) d s,
\end{aligned}
$$

and so, by $\left(H_{4}\right)$,

$$
\left\|\varphi_{0}(t)\right\| \leq M N \int_{0}^{1}\left\|f\left(s, \varphi_{0}(s)\right)\right\| d s<\eta, \quad t \in I .
$$

Since $t \in I$ is arbitrary, we have $\eta>\left\|\varphi_{0}\right\|_{C}=\eta$, a contradiction. Thus (12) is true. Consequently, by the homotopy invariance of the fixed point index, it is easy to get

$$
i\left(A, K \cap B_{\eta}, K\right)=1 .
$$


It follows from (12), (13) and the additivity of the fixed point index that

$$
\begin{aligned}
& i\left(A, K \cap\left(B_{R_{1}} \backslash \bar{B}_{\eta}\right), K\right)=-1, \\
& i\left(A, K \cap\left(B_{\eta} \backslash \bar{B}_{R_{2}}\right), K\right)=1 .
\end{aligned}
$$

Therefore $A$ has at least one fixed point in $K \cap\left(B_{R_{1}} \backslash \bar{B}_{\eta}\right)$ and $K \cap$ $\left(B_{\eta} \backslash \bar{B}_{R_{2}}\right)$, respectively. This completes the proof.

4. Superlinear Sturm-Liouville problems in Banach spaces. In this section we consider the Sturm-Liouville problem:

$$
\left\{\begin{array}{l}
L \varphi=f(t, \varphi), \quad t \in I \\
\alpha_{0} \varphi(0)-\beta_{0} \varphi^{\prime}(0)=\theta \\
\alpha_{1} \varphi(1)+\beta_{1} \varphi^{\prime}(1)=\theta
\end{array}\right.
$$

where $L \varphi \equiv-\left(p(t) \varphi^{\prime}(t)\right)^{\prime}+q(t) \varphi(t), p(t) \in C^{1}(I), p(t)>0, t \in I$, $q(t) \in C(I), q(t) \geq 0, t \in I, \alpha_{i}, \beta_{i}, i=0,1$, are nonnegative constants satisfying $\alpha_{0}^{2}+\beta_{0}^{2}>0, \alpha_{1}^{2}+\beta_{1}^{2}>0$. Assume that 0 is not an eigenvalue of the problem:

$$
\left\{\begin{array}{l}
-\left(p(t) x^{\prime}(t)\right)^{\prime}+q(t) x(t)=\lambda x(t), \quad t \in I, \\
\alpha_{0} x(0)-\beta_{0} x^{\prime}(0)=0 \\
\alpha_{1} x(1)+\beta_{1} x^{\prime}(1)=0
\end{array}\right.
$$

where $x \in C(I)$. Then it is well known that BVP (14) is equivalent to (1) with

$$
k(t, s)= \begin{cases}x(t) y(s) / d & 0 \leq t \leq s \leq 1 \\ x(s) y(t) / d & 0 \leq s \leq t \leq 1\end{cases}
$$

where $d>0$ is a constant and $x, y \in C(I)$ satisfy

$$
\begin{gathered}
-\left(p(t) x^{\prime}(t)\right)^{\prime}+q(t) x(t)=0, \quad x(0)=\beta_{0}, \quad x^{\prime}(0)=\alpha_{0}, \\
-\left(p(t) y^{\prime}(t)\right)^{\prime}+q(t) y(t)=0, \quad y(1)=\beta_{1}, \quad y^{\prime}(1)=-\alpha_{1} .
\end{gathered}
$$

Theorem 3. Let $\beta_{0} \beta_{1}>0$ and $\left(H_{0}\right)$ and $\left(H_{2}\right)$ be satisfied. Suppose that there exists $h \in C(I), h(t)>0$ almost everywhere $t \in I$ such that 
$\left(H_{1}\right)$ is satisfied. Then BVP (14) has at least one positive solution in $C[I, E]$.

Proof. By Lemmas 1-2 in [10] and $\beta_{0} \beta_{1}>0$, we know that $x(t)$ is increasing on $I$ with $x(0)=\beta_{0}>0, y(t)$ is decreasing on $I$ with $y(1)=\beta_{1}>0$. Consequently, $x(t)>0, y(t)>0, t \in I$, i.e., $k(t, s)>0$, $t, s \in I$. Then there exists an $\varepsilon>0$ such that $k(t, s) \geq \varepsilon k(\tau, s)$, $t, s, \tau \in I$. Therefore, Theorem 3 follows from Theorem 1 . This completes the proof.

Example. Consider the infinite system of differential equations:

$$
\begin{cases}-\left(p(t) x_{n}^{\prime}\right)^{\prime}+q(t) x_{n}=h(t) \sum_{i=1}^{\infty}\left|x_{i}\right| / 2^{i} \cdot x_{n} & t \in I, \\ \alpha_{0} x_{n}(0)-\beta_{0} x_{n}^{\prime}(0)=0 & n=1,2, \ldots, \\ \alpha_{1} x_{n}(1)+\beta_{1} x_{n}^{\prime}(1)=0 & \end{cases}
$$

where $h(t) \in C(I)$ with $h(t)>0$ almost everywhere $t \in I$ and $p, q, \alpha_{i}, \beta_{i}, i=0,1$, are as in (14).

Conclusion. If $\beta_{0} \beta_{1}>0$, then system (16) has at least one solution $\left\{x_{n}(t)\right\}$ satisfying $x_{n}(t) \geq 0$ for all $t \in I, n=1,2, \ldots$.

Proof. Let $E=\left\{x=\left(x_{1}, x_{2}, \ldots, x_{n}, \ldots\right)\left|\sum_{i=1}^{\infty}\right| x_{i} \mid / 2^{i}<+\infty\right\}$. Then it is easy to verify that $E$ is a Banach space with norm $\|x\|=$ $\sum_{i=1}^{\infty}\left|x_{i}\right| / 2^{i}$ for $x \in E$. Set $P=\left\{x \in E \mid x_{n} \geq 0, n=1,2, \ldots\right\}$. Clearly $P$ is a normal, reproducing cone in $E$ and system (16) can be regarded as a BVP in $E$ :

$$
\left\{\begin{array}{l}
-\left(p(t) x^{\prime}\right)^{\prime}+q(t) x=f(t, x) \quad t \in I \\
\alpha_{0} x(0)-\beta_{0} x^{\prime}(0)=\theta \\
\alpha_{1} x(1)+\beta_{1} x^{\prime}(1)=\theta
\end{array}\right.
$$

where $x=\left(x_{1}, x_{2}, \ldots, x_{n}, \ldots\right), x^{\prime}=\left(x_{1}^{\prime}, x_{2}^{\prime}, \ldots, x_{n}^{\prime}, \ldots\right)$ and $f=$ $\left(f_{1}, f_{2}, \ldots, f_{n}, \ldots\right)$ with

$$
f_{n}\left(t, x_{1}, x_{2}, \ldots, x_{n}, \ldots\right)=h(t)\|x\| x_{n}, \quad n=1,2,3, \ldots .
$$

It is easy to see that $f$ is uniformly continuous on $I \times\left(P \cap T_{l}\right)$ for any $l>0$, where $T_{l}=\{x \in E \mid\|x\| \leq l\}$. In a similar way as proving the 
examples in $[\mathbf{8}, \mathbf{4}]$, we can show that, for any $D \subset P \cap T_{l}, t \in I, f(t, D)$ is relatively compact in $P$, i.e., $\left(H_{0}\right)$ is satisfied for $f$.

For any $R>0$ sufficiently large, $R_{0}>0$ sufficiently small and for any fixed $b(t) \in C(I)$ with $b(t)>0, t \in I$, it is clear that $f$ satisfies $\left(H_{1}\right)$ and $\left(H_{2}\right)$.

Finally, our conclusion follows from Theorem 3. This completes the proof.

Acknowledgments. The author thanks Professors Dajun Guo and Jingxian Sun for their guidance and encouragement. He is also grateful to the referee for his useful suggestions.

\section{REFERENCES}

1. H. Amann, Fixed point equations and nonlinear eigenvalue problems in ordered Banach spaces, SIAM Rev. 18 (1976), 620-709.

2. K. Deimling, Nonlinear functional analysis, Springer-Verlag, Berlin, 1985.

3. Yihong Du, Nontrivial solutions of superlinear Sturm-Liouville problems, Acta Math. Sinica 35 (1992), 721-728 (in Chinese).

4. Dajun Guo, Existence of solutions of boundary value problems for nonlinear second order impulsive differential equations in Banach spaces, J. Math. Anal. Appl. 181 (1994), 407-421.

5. Dajun Guo and V. Lakshmikantham, Multiple solutions of two-point boundary value problems of ordinary differential equations in Banach spaces, J. Math. Anal. Appl. 129 (1988), 211-222.

6. M.A. Krasnoselskii, New a priori estimates for the solutions of two point boundary value problems and applications, Siberian Math. J. 21 (1980), 85-93.

7. - Topological methods in the theory of nonlinear integral equations, Pergamon Press, 1964.

8. Bendong Lou, Solutions of superlinear Sturm-Liouville problems in Banach spaces, J. Math. Anal. Appl. 201 (1996), 169-179.

9. R.D. Nussbaum, Eigenvectors of nonlinear positive operators and the linear Krein-Rutman theorem, in Fixed point theory, Lecture Notes in Math. 886 (1980), 309-330.

10. Jingxian Sun, Some new fixed point theorems of increasing operators and applications, Appl. Anal. 42 (1991), 263-273.

Department of Mathematics, Shandong University, Jinan 250100, ShanDong, People's Republic of China 